\title{
Autoimmune Encephalitis a Mini Review
}

\author{
Forshing Lui* \\ College of Medicine, California Northstate University, USA \\ *Corresponding author: Forshing Lui, College of Medicine, California Northstate University, USA \\ Submission: 制 February 18, 2019; Published: 觜February 21, 2019
}

\begin{abstract}
Nearly half of all cases of acute encephalitis did not have an identifiable cause. Autoimmune (antibody-mediated) etiology has gained more recognition over the recent years. It is important to consider the diagnosis by the clinical findings of acute limbic encephalopathy, new onset schizophrenia, or new onset medically refractory seizures. Identification of the antibodies is important in defining the underlying causes including underlying malignancies. Antibodies against intracellular proteins are more likely to be paraneoplastic and T-cell mediated. Antibodies against cell surface or synaptic antigens are mostly B-cell mediated and they are more responsive to immunotherapy. In addition to high index of clinical suspicion, MRI, serum and CSF antibody testing are most helpful in making the final diagnosis.
\end{abstract}

\section{Introduction}

Autoimmune encephalitis is a heterogeneous group of inflammatory central nervous system disorders that attracted more medical attention over the past decade. The pathophysiological mechanisms and clinical correlates become better recognized and understood. The entity was very under-recognized in the past and believed to be rare. It occurs in people of all ages, the overall incidence of encephalitis is about 5-8 cases per 100,000 populations yet no cause could be found in nearly half of these cases [1,2]. Autoimmune encephalitis is believed to be the third leading cause of encephalitis after viral encephalitis and acute disseminated encephalomyelitis [1,2]. Early recognition of the diagnosis followed by specific immunological treatment is important in the improvement of the outcome of the patients.

As the diagnosis implies, autoimmune encephalitis is an inflammatory disorder affecting the gray matter or neurons of the CNS. The clinical presentations will include inflammation such as low-grade fever, fatigue, headache and malaise. The predominant clinical features of gray matter involvement will include various forms of seizures, involuntary movements such as dystonia, memory impairment, behavioral or psychotic features and alterations in awareness [2,3]. The most common site of involvement in the CNS is the limbic system especially the medial temporal lobes and hippocampus and hence the common diagnostic term of autoimmune limbic encephalitis. The two most important clinical categories are autoimmune limbic encephalitis and immune epilepsy syndromes with medically refractory seizure as the predominant clinical phenotype.

Autoimmune encephalitis is traditionally classified as paraneoplastic or non-paraneoplastic depending on whether there is any identifiable tumor associated antibodies such as anti-Hu (antineuronal nuclear antibody type 1), anti-Yo, anti Ri and anti$\mathrm{Ma} / \mathrm{Ta}$ etc. [4]. The better and newer classification is to classify the immune encephalopathy according to the targets of the antibodies, whether against intracellular constituents or against cell surface/ synaptic antigens. With this newer and better classification, we understand that the autoimmune encephalitis against intracellular antigens consist of the classical paraneoplastic diseases as listed above as well as others such as anti-GAD (glutamic acid decarboxylase) encephalitis. These diseases are mediated mainly through a predominant T-cell mediated mechanism with cytotoxic $\mathrm{T}$ cells demonstrated in the pathological specimens because the neuronal tissues share similar antigenicity with tumour antigens. These antibodies are generally not pathogenic. On the other hand, the other class of autoimmune encephalitis are immune mediated diseases against cell surface and synaptic antigens such as NMDA (N-Methyl D-Aspartate) Receptor, VGKC (Voltage gated potassium channel) complex, including LGI1 and CASPR2 antibodies. Other antibodies also include AMPA receptor, GABA receptor, mGluR5 receptor and DPPX receptor etc. These are real antibody or B-cell mediated autoimmune encephalitis with real pathogenic antibodies. Some of these are paraneoplastic e.g. anti-NMDAR encephalitis complicating ovarian teratoma in young woman, yet many are not paraneoplastic. These immune encephalitis syndromes often have relatively specific clinical features that may provide good clues to the underlying antibody types. Faciobrachial dystonic seizures is a strong clue to the diagnosis of anti-LGI1 antibody syndrome and confusion, diarrhoea and weight loss will be very suggestive of DPPX antibody syndrome [3,4].

Anti-NMDA receptor antibody limbic encephalitis is worth special mentioning. It is probably the commonest and best characterized type of autoimmune encephalitis or limbic 
encephalitis. It occurs most commonly among young adults and children. The presentation is often very typical and progression predictable. It started with a subacute onset in children and young adults (mean age of around 20) with what appears like a viral syndrome with low grade fever, headache and malaise. This part of the illness is often neglected. The patients will then progress to the next phase of neuropsychiatric presentations of behavioural disturbances and psychosis. Very often, these patients are admitted to inpatient psychiatric units as new onset schizophrenia until they reach the next phase of the illness with speech reduction, refractory seizures, memory impairment, oral and limb dyskinesias, autonomic disturbances (bradycardia, fluctuation in temperatures, salivation and central hypoventilation) and impaired awareness or coma.

Nearly $60 \%$ of young women had an underlying ovarian teratoma [3]. The prognosis may be surprisingly good when diagnosis is recognized early and specific treatment including removal of underlying tumour and immunotherapy instituted appropriately. It is interesting to note that a proportion of patients who present with a late "relapse" of herpes simples' encephalitis is actually suffering from secondary anti-NMDA limbic encephalitis with positive anti-NMDA antibody secondary to the herpes simplex encephalitis [5]. It actually makes real good sense because herpes virus is a neurotropic virus with special predilection to the limbic system with glutaminergic neurons and subsequent formation of anti-NMDA glutamate receptor antibody.

The clue to diagnosis is high index of suspicion. MRI is the most helpful imaging study showing T2 hyperintensity changes involving bilateral more than unilateral temporal lobes [6,7]. Normal MRI, however, does not rule out the diagnosis of autoimmune limbic encephalitis. CSF will show elevated protein with normal glucose and mononuclear cell pleocytosis (between 10-100 cells per cubic $\mathrm{mm}$ ). Both the serum and CSF may show the presence of specific antibodies.

Treatment consists of a two-prong approach: immunotherapy and removal of the immune trigger. If there is an underlying trigger such as a tumour, removal of the tumour is the best option in achieving a cure. Immunotherapy consists of corticosteroid, plasma exchange and intravenous immunoglobulin. If these fail, IV rituximab may be effective by wiping out the B-cells. Most immune encephalitis responds well to immunotherapy while spontaneous remission is very rare [8].

\section{Conclusion}

Autoimmune (antibody mediated) encephalitis is an uncommon yet important cause of acute encephalitis especially limbic encephalitis. It should be considered in any patient presenting with new onset acute encephalitis, refractory seizures or psychosis. Antibodies against intracellular proteins are patho-physiologically different from antibodies against cell surface or synaptic antigens. Diagnosis relies on recognition of specific clinical features, identification of the antibodies in the serum or CSF and aided by MRI findings. Treatment consists mainly of immunotherapy and removal of underlying malignancy if present.

\section{References}

1. Dalmau J, Graus F (2018) Antibody-mediated encephalitis. N EngJ Med 378(9): 840-851.

2. Granerod J, Ambrose HE, Davies NW, Clewley JP, Walsh AL, et al. (2010) Causes of encephalitis and differences in their clinical presentations in England: a multicentre, population-based prospective study. Lancet Infect Dis 10(12): 835-844.

3. Dalmau J, Geis C, Graus F (2017) Autoantibodies to synaptic receptors and neuronal cell surface proteins in autoimmune diseases of the central nervous system. Physiol Rev 97(2): 839-887.

4. Leypoldt F, Armangue T, Dalmau J (2015) Autoimmune encephalopathies. Ann N Y Acad Sci 1338: 94-114.

5. Swayne A, Brown H, Singh G, Blum S (2017) Anti-NMDA receptor encephalitis complicating HSV encephalitis with demonstrated seroconversion. JNNP 88(5): 82.

6. Graus F, Titulaer MJ, Balu R, Benseler S, Bien CG, et al. (2016) A clinical approach to diagnosis of autoimmune encephalitis. The Lancet Neurol 15(4): 391-404

7. Kelly BP, Patel SC, Marin HL, Corrigan JJ, Mitsias PD, et al. (2017) Autoimmune Encephalitis: Pathophysiology and Imaging Review of an Overlooked Diagnosis. Am J Neuroradiol 38(6):1070-1078.

8. Venkatesan A , Tunkel AR, Bloch KC, Lauring AS, Sejvar J, et al. (2013) Case definitions, diagnostic algorithms and priorities in encephalitis: consensus statement of the international encephalitis consortium. Clin Infect Dis 57(8): 1114-1128.
Creative Commons Attribution 4.0 International License

For possible submissions Click Here Submit Article

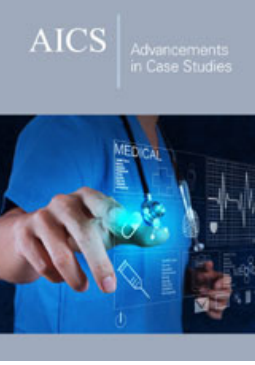

Advancements in Case Studies

\section{Benefits of Publishing with us}

- High-level peer review and editorial services

- Freely accessible online immediately upon publication

- Authors retain the copyright to their work

- Licensing it under a Creative Commons license

- Visibility through different online platforms 